\title{
Development of 3D Concrete Extrusion Nozzle for Producing Free-form Concrete Panels
}

\author{
Ji-Yeong, Yun ${ }^{1}$, Kyeong-Tae, Jeong ${ }^{2}$, Donghoon, Lee ${ }^{* 3}$ \\ 12 Master's course, Department of Architectural engineering, Hanbat National University, Daejeon, 34158, \\ Republic of Korea \\ *3 Professor, Department of Architectural engineering, Hanbat National University, Daejeon, 34158, Republic \\ of Korea \\ *Corresponding author. E-mail: donghoon@hanbat.ac.kr
}

Article History:Received:11 november 2020; Accepted: 27 December 2020; Published online: 5 April 2021

Abstract: There has been an increase in demand for free-form building through the development of advanced technologies, and the fourth industrial revolution has become a worldwide trend, thereby changing the construction industry. In particular, in the case of the free-form architecture sector, development of 3D printing technologies has been ongoing for construction automation. According to such trends, this study develops an FCP production equipment using 3D printing technologies. The FCP production equipment in this study is made up of mould equipment and 3D printer. It is different from existing 3D printing technologies so in this study 3D concrete extrusion nozzle must be developed for producing FCP. Basic design suitable to such requirements is proposed. Applicability of the proposed design is checked and the nozzle form is concretized to draft the final drawing. In this study, slit-type opening and closing device for accurate extrusion stoppage of concrete and screw-type nozzle for adjusting pressure and extrusion speed were applied for the nozzle. This is expected to be innovative technology for the FCP production sector.

Keywords: Construction 3D printing, Construction automation, Free-form building, Free-form concrete panel, Concrete extrusion nozzle

\section{Introduction}

The development of advanced technologies has enhanced design freedom, while also resulting in growing demand for free-form construction[1]. Free-form construction displays the technological and economical superiority of countries and cities, and serves as a new scale containing socio-cultural meaning[2]. In addition, the fourth industrial revolution has become a global trend and is transforming the construction industry. Technologies such as 3D printing, construction robotics, smart construction, etc. are continuously being developed for construction automation. Various attempts are also being made in the free-form construction sector to perfectly implement building using automation technologies. But there is still a lack of elemental technologies related to free-form building and therefore, enormous amounts of capital are being injected for construction. Particularly in the case of free-form construction panel (FCP) production, various technologies are being developed, but there are issues such as that moulds cannot be reused for producing panels, while also having issues related to time and cost. For example, the FCP that makes up the exterior of the Qatar National Museum has different shapes and curves. Therefore, it raises production costs and wages because the number of moulds that must be produced increases due to the diversity of panels. Furthermore, this increases the amount of wastes[3]. Such problems result in the need of massive amounts of capital, therefore lowering the productivity of free-form building. New automation technologies must be developed for producing FCP in order to solve such issue.

Therefore, the authors of this study intend to develop FCP production equipment utilizing 3D printing technologies. Prior to development of the equipment, this study develops the core technology for 3D printing equipment for producing FCPs, which is the concrete extrusion nozzle. The development process of the nozzle is as shown in Figure 1.

*Corresponding author: Donghoon, Lee

Professor, Department of Architectural engineering, Hanbat National University, Daejeon, 34158, Republic of

Korea. E-mail: donghoon@ @anbat.ac.kr 


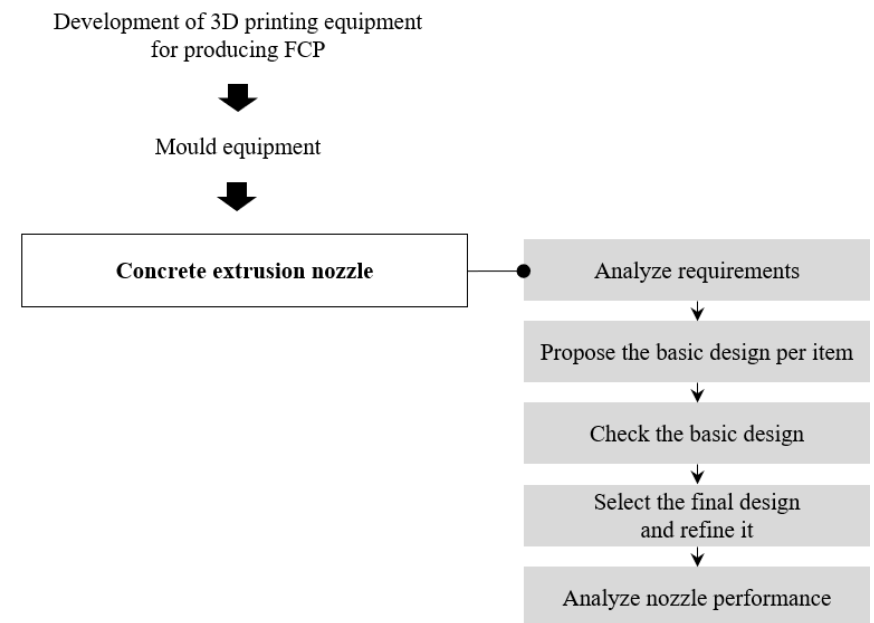

Figure 1. Process of developing nozzle

In order to develop the nozzle, the nozzle requirements are analyzed and the basic design per item is proposed. After checking whether the presented nozzle can be applied as the nozzle, the final design of the nozzle is selected. Afterwards, the detailed drawings of the nozzle are drafted. Lastly, the nozzle performance is analyzed to check whether the requirements are satisfied.

\section{Literature review}

\subsection{Free-form concrete panel production technology}

The free-form concrete members of the Dongdaemun Design Plaza used wooden moulds. Like traditional methods, it produced a free-form mould using wood to produce free-form concrete members. This method allows free-form moulding, but cannot be reused, thus raising construction time and wages, and there is risk of error due to manual work[4].

Fiber moulds were developed to resolve the weakness of wooden moulds[5]. Unlike existing mould methods, fiber moulds are very flexible when making panels having complex forms. But as shown in Figure 2 , fiber moulds have a difference in thickness in the cross-section, and therefore, errors can occur during the construction of free-form building[6].
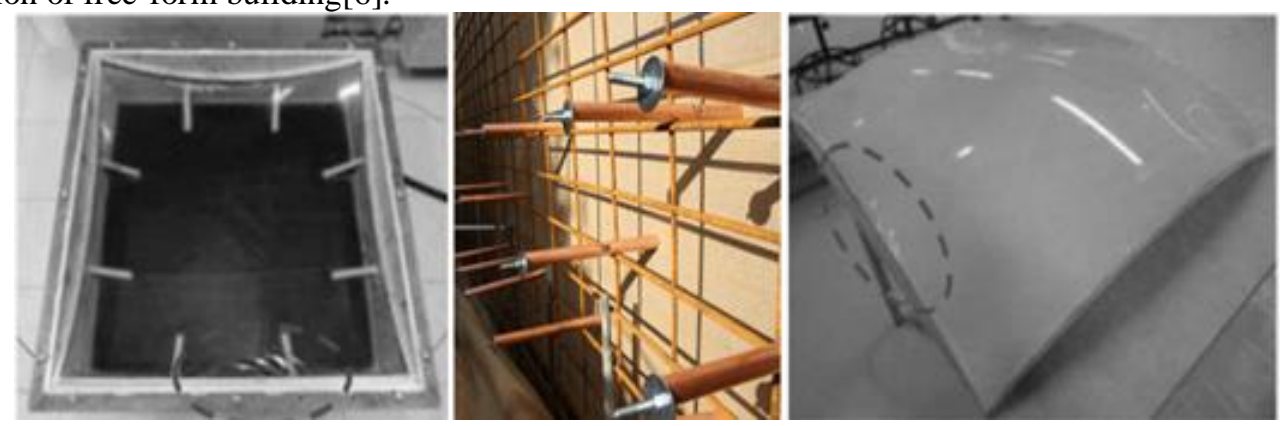

Figure 2. Fiber mould[5]

In order to reduce errors in the production of free-form panels, a 3D processing machine was developed to machine EPS into mould as shown in Figure 3[7]. This can reduce manpower and costs by automating production. However, its processing precision is not superior to CNC milling machines for which the precision does not exceed $1 \mathrm{~mm}$, and therefore, errors can occur when producing panels. In addition, the processed EPS mould cannot be reused.
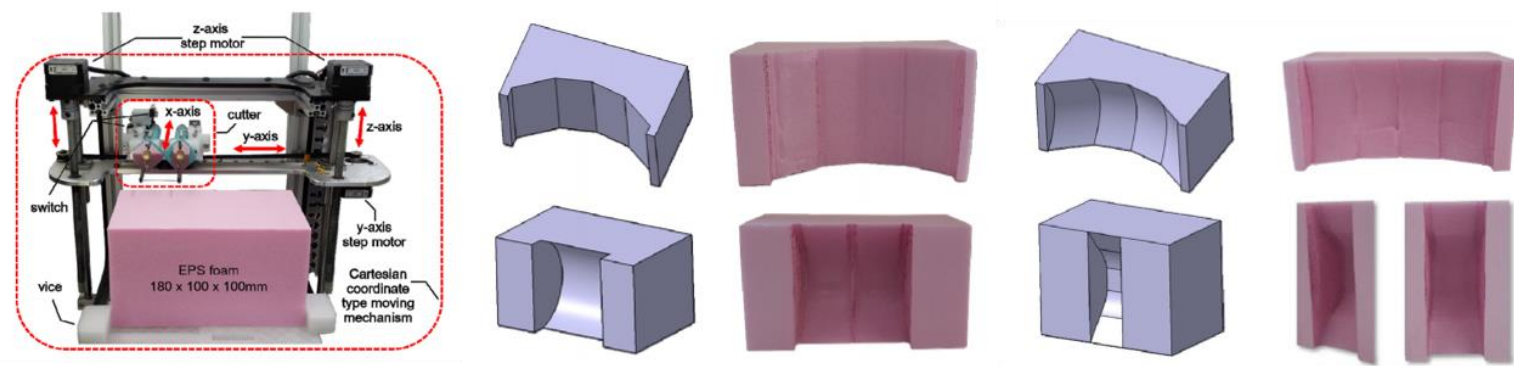

Figure 3. EPS mould[7] 
A wax mould was developed as shown in Figure 4 to resolve the issue of reusing moulds[8,9]. [8] simply presented the concept of a wax mould and [9] developed reusable PCM manufacturing technologies using CNC machines. However, this technology requires a considerably long time for producing moulds and the wax can melt due to the hydration heat generated during curing, thus making it difficult to create a smooth form for the panel.

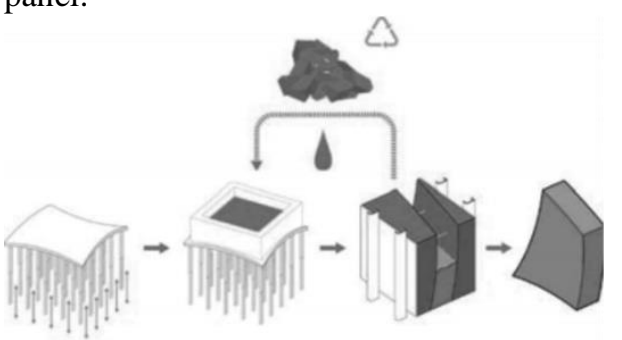

(a) Wax mould

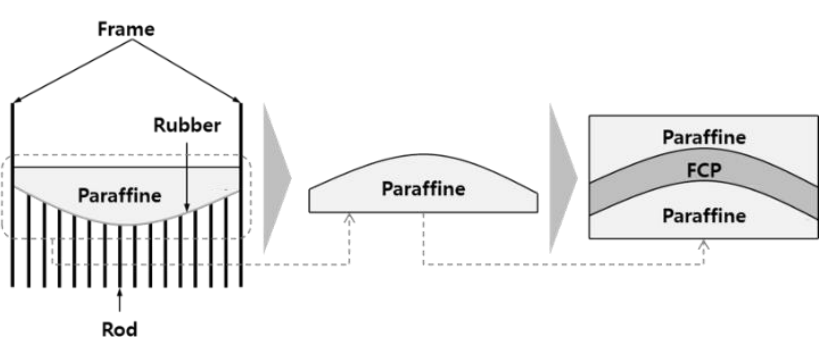

(b) PCM mould

Figure 4. Wax mould[8,9]

\subsection{Construction 3D printing technology}

Construction 3D printing began in 2004 at the University of Southern California with contour crafting technologies. CRAFT (Center for Rapid Automated Fabrication Technologies) conducted studies on the production and utilization of large machines for construction automation. Construction 3D printing technologies spray concrete to form a structure. This construction automation method can reduce labor, time and cost significantly. Starting with this technology, many countries around the world have been continuously developing 3D printing technologies for construction.

CyBe Construction of the Netherlands mounted a concrete extrusion nozzle on a robot arm to develop 3D printing equipment. A caterpillar was mounted on the robot arm to improve equipment operation efficiency. $\mathrm{CyBe}$ Construction now possesses many construction cases such as completing the single-story architecture of R\&Drone Laboratory (2017), 3D STUDIO 2030 (2018), etc. in the United Arab Emirates[10].

IMCRC (Innovative Manufacturing and Construction Research Center) of Loughborough University in England conducted research on free-form concrete production 3D printing technologies[11]. Among the moulds of this technology, the master mould was produced using 3D printing methods to produce a clear shape in the mould. This technology requires additional processes and it takes a long time to produce panels, and therefore, mass production is not possible[12].

\section{Concept of 3D printing equipment for producing FCP}

This study develops a concrete extrusion nozzle for 3D printing equipment for producing FCPs. The 3D printing equipment for producing FCP is comprised of the mould equipment and 3D printer as shown in Figure 5. First, the mould equipment is comprised of side-shaped control equipment, variable side mould, and lower multi-point press equipment. This equipment can be produced freely with various shapes of the FCP. The 3D printer is comprised of the nozzle and frame, and it is a device that extrudes concrete into the mould equipment.

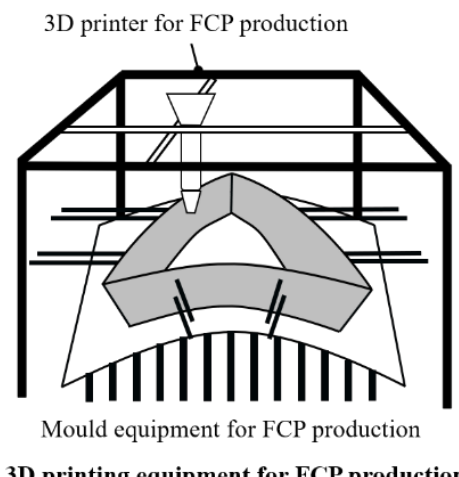

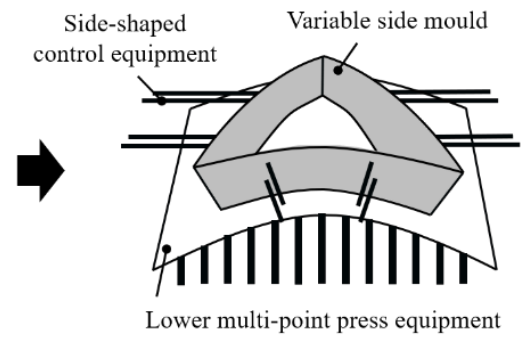

Mould equipment

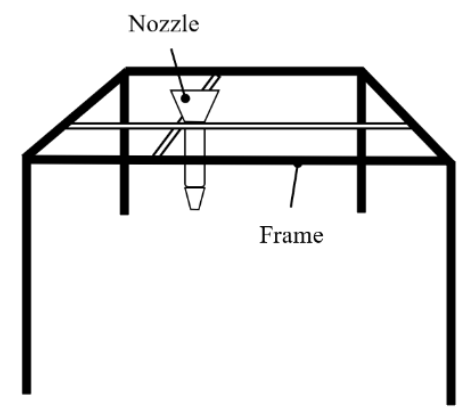

3D printer

Figure 5. Composition of the 3D printing equipment for producing FCP

The FCP production process of this equipment is as shown in Figure 6. First, the side-shaped control equipment, variable side mould, and lower multi-point press equipment are combined to fit the FCP form to make the mould. A specified amount of concrete is extruded into the $3 \mathrm{D}$ printer within the combined mould and cured it. Lastly, once curing ends, the mould is disassembled to complete production of FCP. 


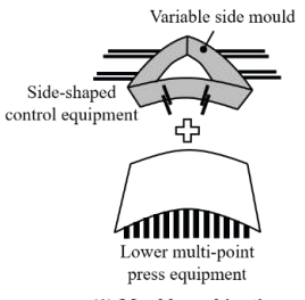

(1) Mould combinatio

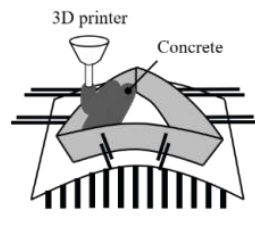

(2) Concrete extrusion

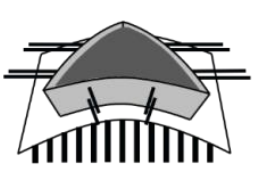

(3) Concrete curing

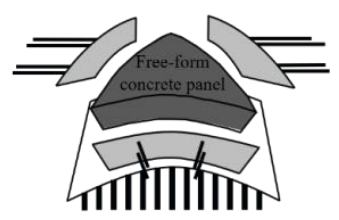

(4) Demould

Figure 6. Process for producing FCP

Mold equipment is implemented in the form of a panel during the FCP production process. As shown in Figure 7, this study developed the mould equipment having a method of fastening the side mould form of the panel through movement of the side rods arranged in the side shape control equipment. At this time, the variable side mould forms a curve on the side of the panel and rods arranged on the lower multi-point press equipment [9] form the curves on the bottom of the panel. This equipment can freely implement moulds of various forms as needed for the panel and the shape can be adjusted automatically.
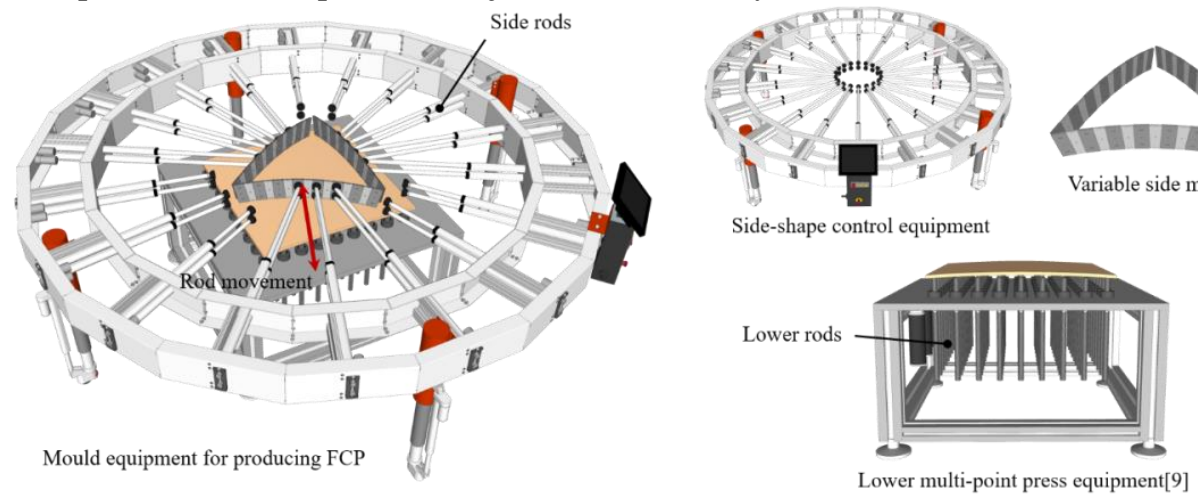

Figure 7. Mould equipment for producing FCP[1]

FCP cannot be produced with only the movement of 3D printers. Unlike existing construction 3D printing technologies that pile vertically only, 3D printing technologies used for producing FCP can pile concrete at a slope to form a curve for the panel. When loading mortar or concrete at a slope, it can collapse before hardening completing due to the properties of the materials. Therefore, lower and side mould equipment are essential to maintain the form of the FCP as shown in Figure 7.

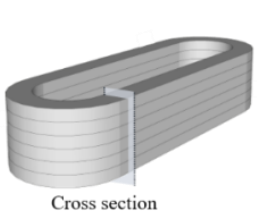

Existing 3D printing technology for construction

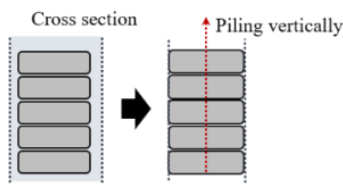

Figure 8. Piling form of 3D printing

The need for moulds can also be checked in the nozzle path as shown in Figure 9. Existing 3D printing technologies for construction forms a wall by extruding concrete on two dimensions (x-y). But 3D printing technologies for producing FCP do not only move the nozzle at the same level. This movement occurs simultaneously in the plane (x-y) and top-down (z). In other words, because the nozzle moves in three dimensions (x-y-z), mould equipment that can maintain the form of the concrete extruded into the nozzle is essential.

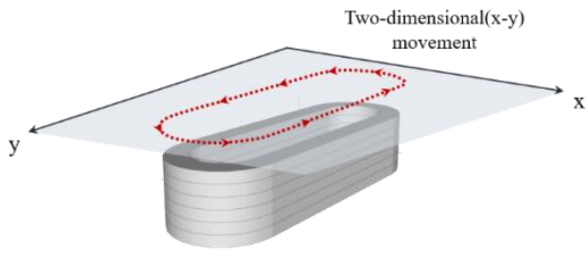

Existing 3D printing technology for construction

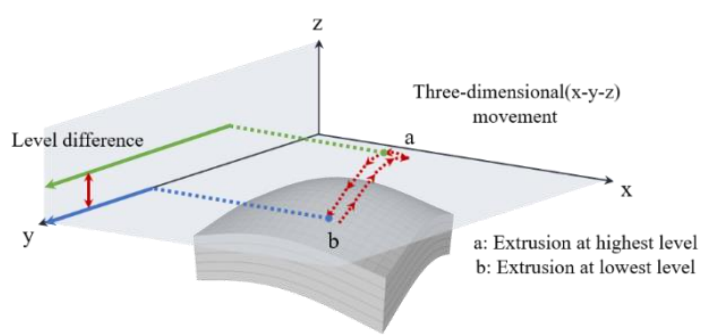

$3 \mathrm{D}$ printing technology for $\mathrm{FCP}$ production

Figure 9. Path of nozzle

Molds require precision in concrete extrusion. The FCP production nozzle must precisely extrude a specific 
amount of concrete within the mould having a certain shape. Currently used construction 3D printing technologies form the wall by having the nozzle continuously extrude concrete. At this time, the nozzle cannot stop the extruded concrete precisely. Due to the strong pressure of concrete emitted from the pump, the concrete spouts or flows out without accuracy. When using such nozzle, it is impossible to adjust the specific amount of extrusion and FCP cannot be shaped completely. This has a huge impact on the quality of the panel and would require rework, thus raising time and cost. It is therefore necessary to develop a new nozzle suitable to the 3D printing equipment for producing FCP.

\section{Development of nozzle for 3D printer}

\subsection{Requirements of nozzle}

FCP production nozzles have moulds with a specific shape and thus requires precision of concrete, and therefore, it is necessary to adjust concrete extrusion. Unlike existing nozzle forms, precise extrusion requires extrusion stop performance. That is why opening and closing device that can stop extrusion quickly and precisely for the nozzle. But when using opening and closing device, concrete is leaked as shown in Figure 10. When the extrusion outlet is blocked, the concrete left in the nozzle leaks from the gap between the opening and closing device and nozzle caused by pressure. This makes it difficult to produce FCP precisely. Therefore, the nozzle must be able to disperse pressure that occurs inside the nozzle to prevent leaking of concrete.

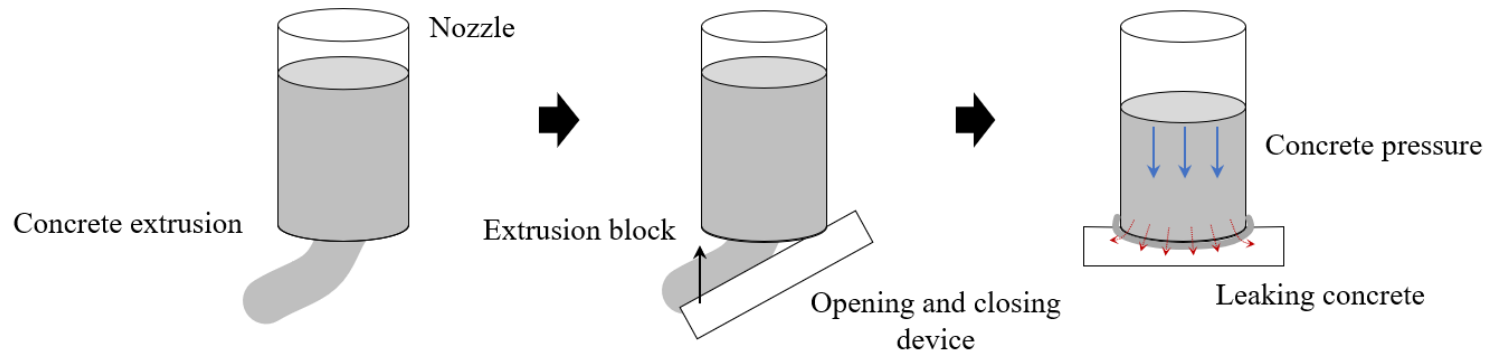

Figure 10. Leakage of concrete

The nozzle must extrude concrete precisely in the mould, and therefore, extrusion speed of the nozzle must be adjusted. For example, the concrete extrusion zone is divided into the central and edge parts as shown in Figure 11 (a). Unlike the central part, the edges come into contact with the mould, and thus, smooth surface treatment of the panel is needed during concrete extrusion. In other words, it is fine for the central part of the mould to extrude concrete quickly, but concrete should be extruded slowly for precise extrusion in the edges. After completing concrete extrusion, FCP should be able to adjust concrete extrusion quantity for smooth finishing. As shown in Figure 11 (b), concrete is extruded in the upper part of the FCP to form a smooth surface. The nozzle used here must be able to extrude small amounts of concrete. Therefore, it is necessary to be able to adjust the nozzle pipe diameter to regulate extrusion amount. Also, adjusting pipe diameter of the nozzle is crucial for producing different types of panels. In the case of panels with small sizes or panels with small angles that make up the mould, precise extrusion of the panel is required. That is why it is essential to be able to adjust the pipe diameter of the nozzle.

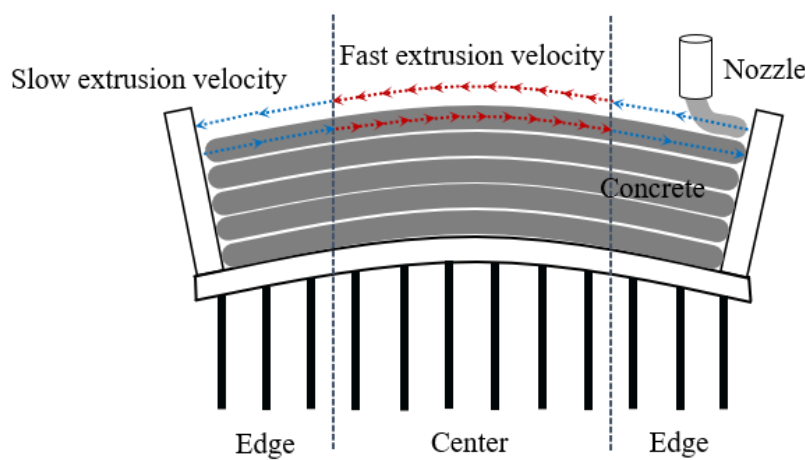

(a) Extrusion velocity control

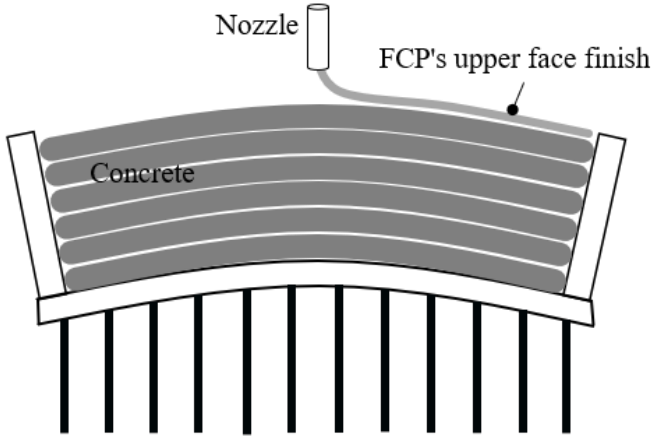

(a) Extrusion volume control

Figure 11. Adjust the speed and amount of concrete extrusion

\subsection{Basic design of nozzle by requirements}

The concrete extrusion nozzle developed in this study must satisfy the following requirements. Opening and closing device that can stop concrete extrusion accurately is needed and it must be able to disperse pressure that occurs when blocking the extrusion outlet. Extrusion speed must be adjustable to ensure precise extrusion of 
concrete, and the pipe diameter of the nozzle must be adjustable to produce various panels and for the finishing of the top of the FCP. In order to develop a nozzle that satisfies these four items, the basic design was first presented.

The opening and closing nozzle is divided into rotating type and slit type as shown in Figure 12. Circular plate type is that the plate on the ends of the nozzle rotate by $90^{\circ}$ to open and close the nozzle entrance. Ball type opens and closes the nozzle pipe by rotating a ball inside the nozzle. Slit type opens and closes the nozzle pipe by having a rectangular slit move left and right inside the fixed slit frame.

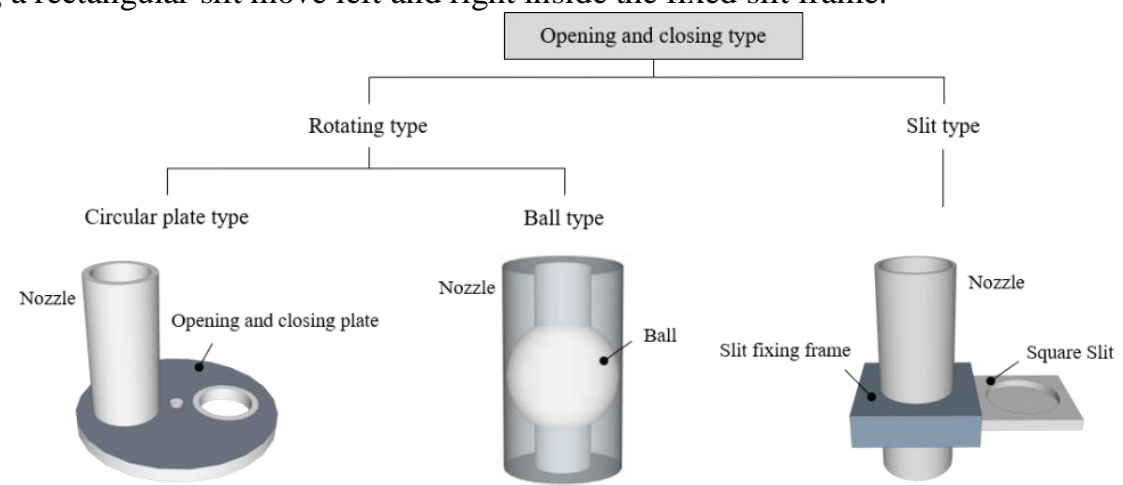

Figure 12. Nozzle design in opening and closing type

The pressure adjusting nozzle is divided into the cylinder-type and screw type as shown in Figure 13. The cylinder type nozzles, one way type and two ways type, use the principle of pistons to disperse concrete pressure. The screw type nozzle reverses the motor to adjust the pressure.

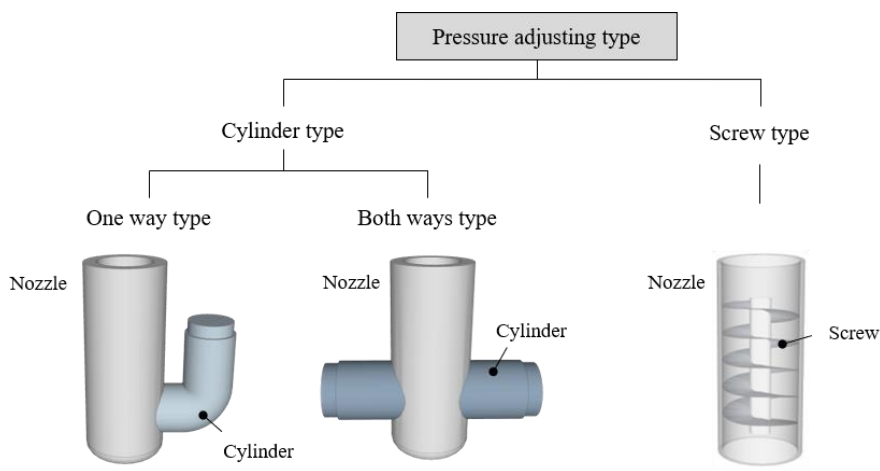

Figure 13. Nozzle design in pressure adjusting type

The nozzle does not extrude concrete with the pump pressure. The pump extrudes concrete continuously and therefore cannot adjust extrusion speed. Precise extrusion is not possible using the pump and is therefore inappropriate for the nozzle for producing FCPs. Therefore, a screw type nozzle is used to adjust extrusion speed. This extrudes concrete using the rotation of the motor and because the extrusion speed changes according to the number of rotations, the extrusion speed can be adjusted. When using the screw type without using a pump, a tank that can temporarily store concrete is needed.

The nozzle pipe cannot be replaced for each size needed when adjusting the pipe diameter of the nozzle. Therefore, in order for the nozzle to automatically replace the size of the pipe diameter, it is designed to make pipe diameter adjustable in the opening and closing device as shown in Figure 14. The pipe diameter of the nozzle is maintained constantly and the size of the nozzle entrance is adjusted in the bottom of the opening and closing device. In other words, the method for adjusting the nozzle's pipe diameter can change depending on the form of the opening and closing device.

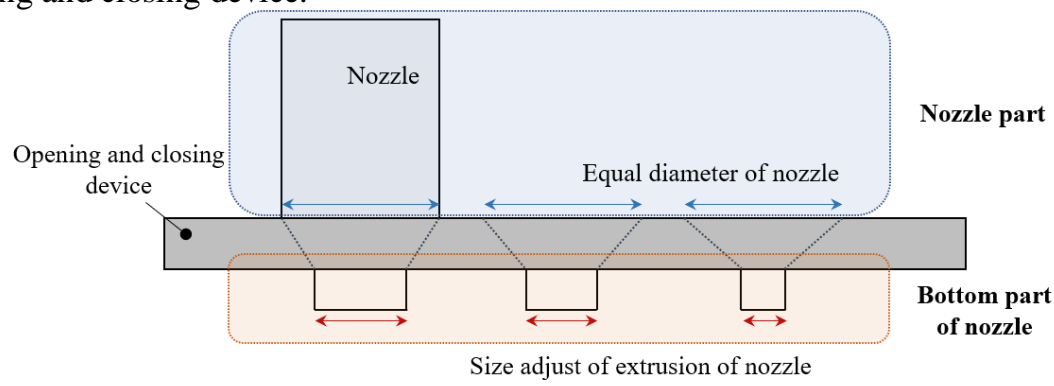

Figure 14. The nozzle pipe diameter adjustment method 


\subsection{Analyze design of nozzle}

In order to judge whether the above design is suitable for the nozzle to be developed in this study, nozzle design is analyzed. It must be verified whether the design is mechanically possible and whether the nozzle is produced efficiently according to requirements. In addition, possibility of contamination of the equipment due to the concrete used as the main ingredient and whether it is easy to clean and replace the nozzle production part, which is not permanent, must be verified. Thus, the opening and closing type nozzle and pressure adjusting type nozzle design was analyzed based on the four standards of mechanical configuration, efficiency, possibility of flaws, and cleaning and maintenance.

Opening and closing type nozzles are comprised of rotating and slit types. And they are analyzed as shown in Table 1. For ball type, when the ball rotates, concrete flows in between the ball and nozzle. And for slit type, concrete also flows inside between the slit and slit frame, thus having high possibility for causing mechanical flaws. Therefore, cleaning becomes difficult and malfunctions can occur easily. Circular plate type has a large area for the opening and closing device and so when the nozzle moves, it can destroy the form of unhardened concrete. Therefore, it is inefficient as it must take into consideration the movement of the nozzle for each panel type. However, mechanical configuration and cleaning is easy and there is low possibility for flaws.

Table 1. opening and closing type nozzle design analyze

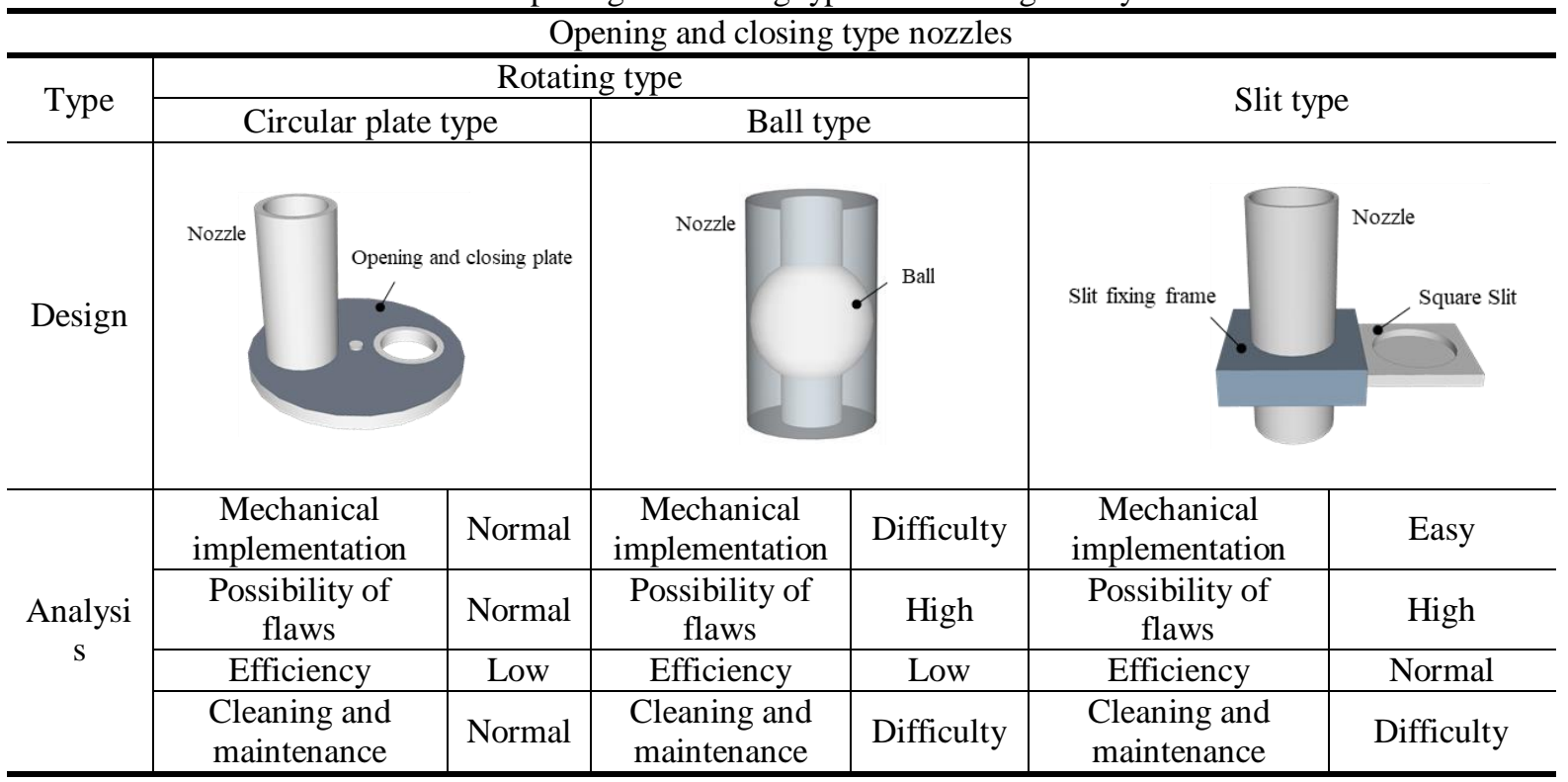

The design of the pressure adjusting type nozzle is analyzed as shown in Table 2. It is difficult to implement cylinder activation method for the cylinder types, and concrete may flow through the gaps in the cylinder, therefore making it possible for mechanical defects to occur. It is thus difficult to clean and maintain. Meanwhile, the screw type nozzle activates through inverse rotation of the motor, and therefore, it is easy to implement. Because screw rotation is constant, there is low possibility for flaws caused by concrete and cleaning and maintenance is also easy. In addition, both pressure and extrusion speed can be regulated for screws, making it more efficient.

Table 2. Pressure adjusting type nozzle design analyze

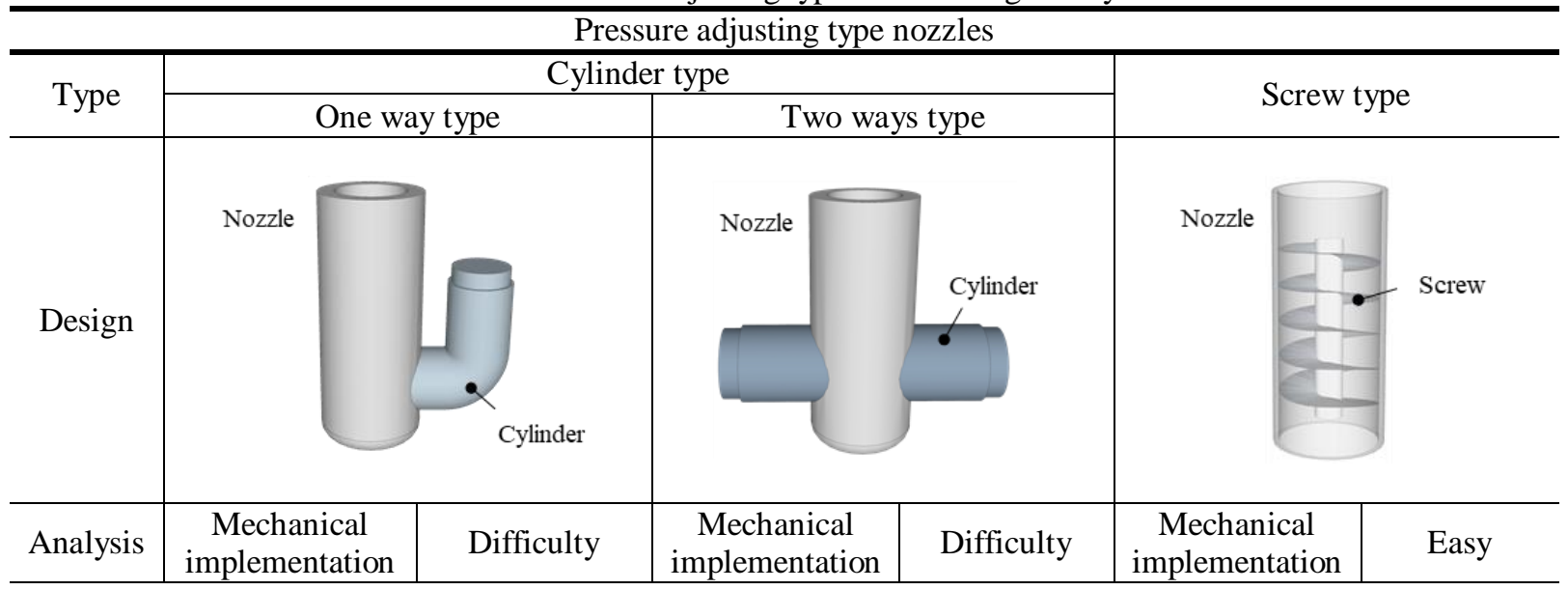




\begin{tabular}{c|c|c|c|c|c|c}
\hline & $\begin{array}{c}\text { Possibility of } \\
\text { flaws }\end{array}$ & High & $\begin{array}{c}\text { Possibility of } \\
\text { flaws }\end{array}$ & High & $\begin{array}{c}\text { Possibility of } \\
\text { flaws }\end{array}$ & Low \\
\cline { 2 - 6 } & Efficiency & Normal & Efficiency & Normal & Efficiency & High \\
\cline { 2 - 7 } & $\begin{array}{c}\text { Cleaning and } \\
\text { maintenance }\end{array}$ & Difficulty & $\begin{array}{c}\text { Cleaning and } \\
\text { maintenance }\end{array}$ & Difficulty & $\begin{array}{c}\text { Cleaning and } \\
\text { maintenance }\end{array}$ & Normal \\
\hline
\end{tabular}

\subsection{Final design of nozzle}

The nozzle design that satisfies requirements was concretized based on the results of analyzing basic designs for selecting the final design. As shown in Figure 15, the first design selected the rotating type opening and closing device and dual nozzle method. This device was designed by modifying the form of the circular plate type in Figure 12. The circular opening and closing device is inefficient as it can destroy the form of the concrete. In order to resolve this, the rotating axis of the opening and closing device is slanted and designed to prevent interference of the concrete form. However, this method places the rotation axis of the opening and closing device at a slope, therefore making mechanical implementation difficult. In addition, when the opening and closing device rotates, areas where concrete cannot be blocked off will occur, thus causing concrete to flow out.

To solve this, a fan-shaped device was designed instead of a circular shape in the second design. The fanshaped form minimizes the necessary area for the opening and closing device, and therefore does not interfere with extruded concrete. Furthermore, mechanical implementation is simple as it can design the nozzle's axis and rotating axis parallel. The nozzle pipe diameter is adjusted in the bottom of the opening and closing device as explained earlier. First, it is important to select the format of the opening and closing device. It was thus judged that the dual nozzle form is a secondary requirement, and so the nozzle pipe diameter adjustment method was changed to a single nozzle. The dual nozzle was designed to allow easy assembly and disassembly of the opening and closing device for easy changes after completing development of the nozzle.

The nozzle developed in this study must be able to extrude concrete with precision. Precise extrusion requires not only adjustment of extrusion quantity and speed, but also precise stoppage of extrusion. The nozzle needs opening and closing device that can accurately stop extrusion of concrete. In addition, a mechanical implementation method that can move the nozzle constantly and at a strong force is needed, and it must be possible to arrange this efficiently. A fan-shaped rotating opening and closing device with selected in the second design makes precise extrusion possible. But it is difficult to fasten the motor that rotates the opening and closing device, and when placing this close to the nozzle part, there is the risk that water can glow into the motor when cleaning the nozzle, thus causing malfunctions. For such reason, the slit type was selected over rotating type.

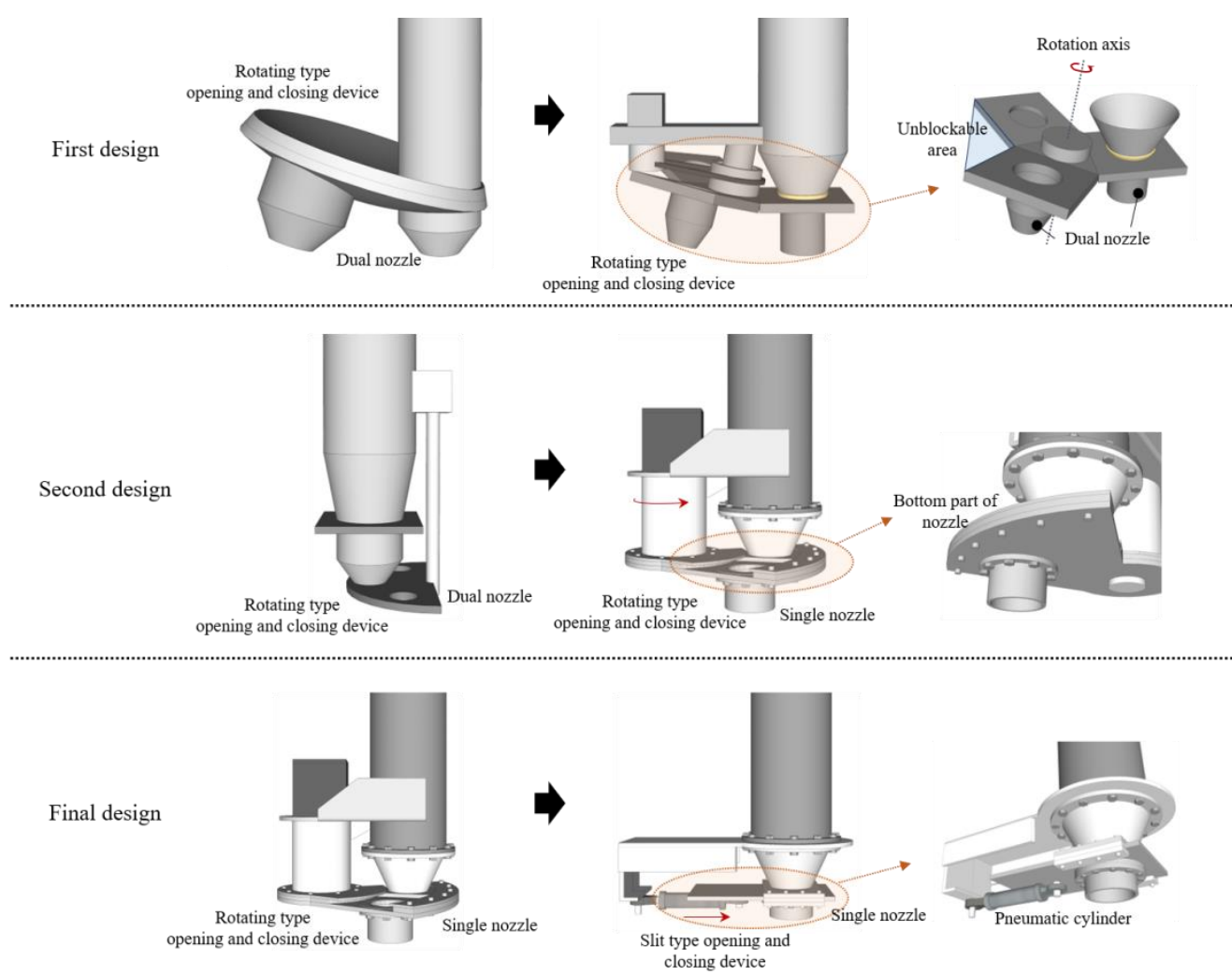

Figure 15. Final design of nozzle selection 


\subsection{Analyze performance of nozzle}

The performance of the nozzle was analyzed to check whether the requirements of the finally selected design were satisfied as shown in Figure 16. The nozzle is mainly made up of the nozzle part and open and close part. First, the nozzle part is comprised of the mixing tank and screw nozzle. Because no pump is used, concrete must be placed in a mixing tank in advance. The screw type nozzle can adjust the required extrusion speed and pressure. This allows precise extrusion and when blocking the extrusion outlet of the nozzle by adjusting pressure through inverse rotation of the motor, it can prevent concrete from leaking. The open and close part is made up of the opening and closing device and extrusion outlet. The opening and closing device is a slit type and can stop concrete extrusion using a simple method. This is implemented through the movement of a pneumatic cylinder. The extrusion outlet on the bottom of the opening and closing device is a single nozzle rather than a dual nozzle. Adjustment of the nozzle pipe diameter changes according to the type of opening and closing device, and therefore, it is necessary to first develop this device. Thus, the single nozzle was selected and in order to easily change the form of the nozzle once development of the opening and closing device is completed, the bolt connection method was applied for easy assembly and disassembly of the nozzle. This method allows easy cleaning with water and easy replacement when having to replace parts because of contamination from materials.

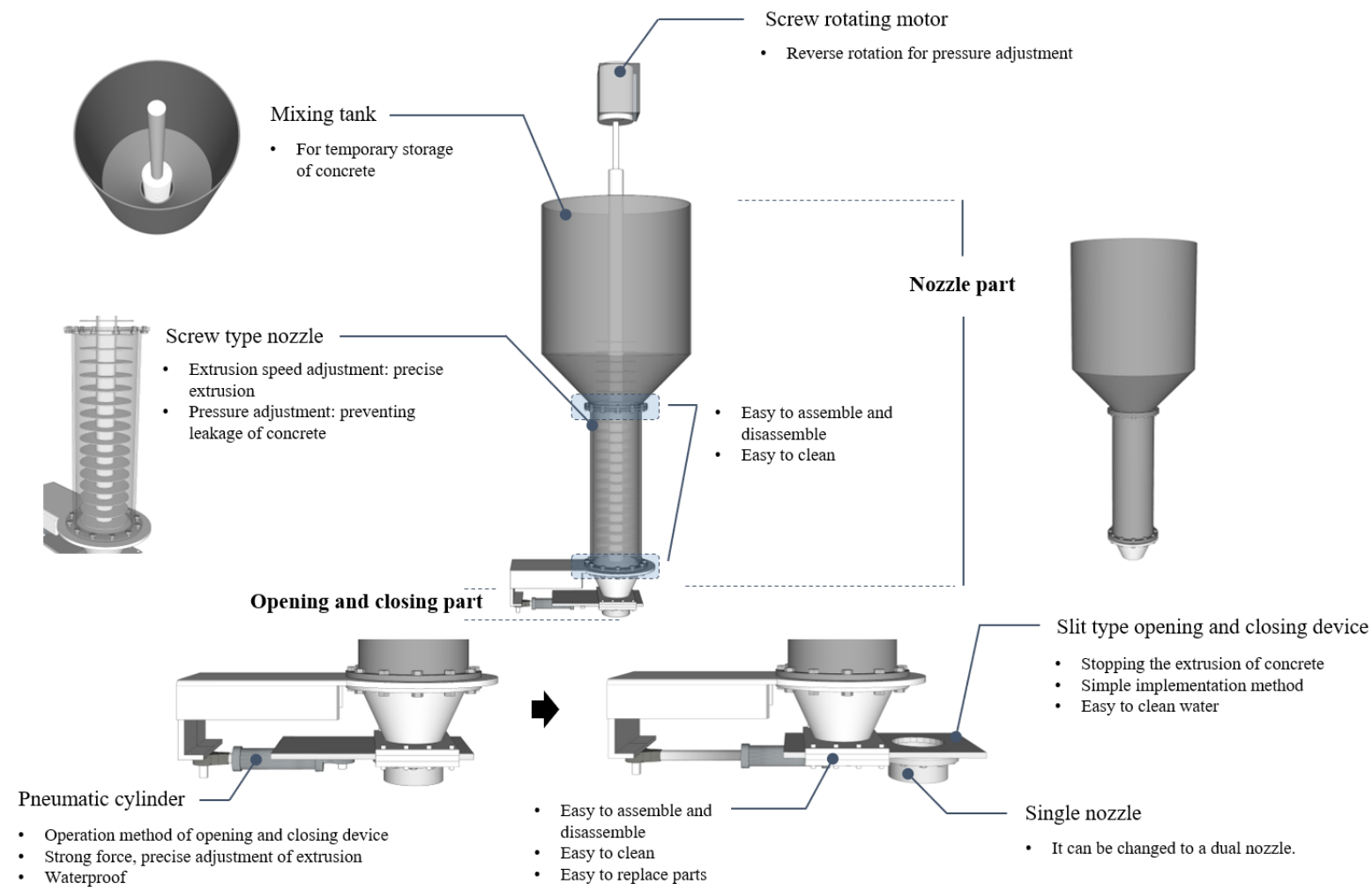

Figure 16. Performance analyze of nozzle

\section{Conclusion}

This study developed an FCP production equipment using 3D printing technologies to resolve problems of FCP. In this study, the core technology of 3D printing equipment, which is concrete extrusion nozzle, was developed. Unlike existing 3D printing technologies for construction, this technology is on producing FCP. The requirements was analyzed for developing a new nozzle. The basic design per item was proposed based on the nozzle requirements. Standards were set to judge whether the presented design is suitable for the nozzle being developed in this study, and the design was analyzed. The final design was selected based on the analysis results. For the selection, slit type opening and closing device for accurate extrusion stoppage of concrete and screw type nozzle for adjusting pressure and extrusion speed were applied. Performance was analyzed to judge whether the selected design satisfies the requirements of the nozzle. The nozzle satisfied the requirements for opening and closing device and adjusting pressure and extrusion speed, but the nozzle's pipe diameter adjustment method applied single nozzle, and therefore, did not satisfy requirements. It is because the nozzle pipe diameter adjustment method changes according to the type of the opening and closing device. The dual nozzle can be applied after completing development of the nozzle.

In this study, concrete extrusion nozzle was developed. But this is a nozzle for producing FCP and it was developed to produce panels by extruding concrete into the mould equipment. Thus, it cannot be applied in 
general 3D printing technologies for construction, and can only be applied in the FCP production equipment developed in this study. This study is a basic study on 3D printing technologies for FCP production, and additional concrete extrusion tests to accurately analyze nozzle performance is necessary. Through this, by analyzing the nozzle performance and supplementing the technologies applied to the nozzle to implement FCP production 3D printing technology, it is expected that it will be possible to resolve the issues of FCPs. Moreover, this is expected to be innovative technology for the FCP production sector.

\section{Acknowledgements}

$\checkmark \quad$ This work was supported by the National Research Foundation of Korea(NRF) grant funded by the Korea government(MSIT) (No. 2020R1C1C1012600).

$\checkmark \quad$ This work is supported by the Korea Agency for Infrastructure Technology Advancement(KAIA) grant funded by the Ministry of Land, Infrastructure and Transport (Grant 20CTAP-C151959-02).

\section{References}

1. K. T. Jeong, J. Y. Yun, K. H. Kim, D. Lee. (2020) Development of Operation Technology and TwoSided Multi-Point Press Equipment for Improving Accuracy of FCP. Journal of Test Engineering \& Management. 83vol:4222-4233.

2. K. Lee. (2008) Construction Issues of Irregular-Shaped Buildings. Architectural institute of korea. 52(4):63-65.

3. D. Lee, D. B. Jang, S. Kim. (2014) Production Technology of Free-form Concrete Segments using Phase Change Material. International Conference on Advances In Civil and Structural Engineering. CSE 2014, Kuala Lumpur, Malaysia. 45-48.

4. H. H. Kim, E. C. Kang. (2010) Dongdaemun Design Plaza \& Park. Magazine of the Korea Concrete Institute. 22(5):100-104.

5. R.W.A. Verhaegh. (2010) Free forms in concrete: the fabrication of free-form concrete segments using fabric formwork [master's thesis]. Eindhoven University of Technology. 153 p.

6. K. Kim, C. Lim, S. Kim. (2013) A basic study of manufacturing technology of free-form concrete segments. Conference of the Korea Institute of Building Construction. 13(1):12-13.

7. J. H. Seo, D. H. Hong. (2017) 3D Cutting Machine of EPS Foam for Manufacturing Free-Formed Concrete Mold. Journal of the Korean Society for Precision Engineering. 34(1):35-39.

8. S. Oesterle1, A. Vansteenkistel, A. Mirjan1. (2012) Zero Waste Free-Form Formwork. Proceedings of the Second International Conference on Flexible Formwork. Icff 2012 - Second International Conference on flexible Formwork, Bath, UK. 2012 258-267.

9. D. Lee. (2015) A Study of Construction and Management Technology of Free-form Buildings [master's thesis]. Kyung Hee University. 186 p.

10. S. K. Yoo, H. S. Park, S. C. Bae. (2019) A Case Study of Domestic and Oversea Concrete 3D Printing Technology and Applications. Magazine of the Korea Concrete Institute. 31(1):58-64.

11. Innovative materials developed for construction industry [Internet]. (2011) [updated 2012 Oct 25].

12. K. Kim, K. Son, E. D. Kim, S. Kim. (2014) Current trends and future directions of free-form building technology. Journal of Architectural Science Review. 58(3):230-243. 